\title{
Evaluation of 25 years of phototherapy for treating psoriasis at a teaching hospital in southern Spain*
}

\author{
Tomas Toledo-Pastrana ${ }^{1}$ \\ Ana María Carrizosa-Esquivel ${ }^{3}$
}

\author{
María José García-Hernández ${ }^{2}$ \\ Francisco Miguel Camacho-Martínez ${ }^{3}$
}

DOI: http:/ /dx.doi.org/10.1590/abd1806-4841.20153694

\begin{abstract}
BACKGROUND: For years, phototherapy has been used in a wide range of skin diseases, which is unsurprising as skin is the anatomical feature most directly exposed to light, especially in psoriasis. Although the role of light therapy has been replaced by different therapeutic modalities in recent years, this treatment is now an established option for many skin diseases.

ОвјестіVEs: The aim was to characterize the patient population thathad received the aforementioned treatment in the Virgen Macarena Health Area in Seville (Spain) between June 1985 and October 2011.

MetHods: We have designed a descriptive study with a univariate analysis covering 443 treatments with light therapy, all administered to the same number of patients suffering from psoriasis.

RESULTS: $79.15 \%$ of patients were discharged due to improvement or healing, while the $20.85 \%$ were discharged due to other reasons. The average total accumulative dose was $131.53 \mathrm{~J} / \mathrm{cm} 2$. We do not detected an increase in proportion in patients for develop NMSK after light therapy treatment.

ConcLusion: We consider that phototherapy is still an effective and efficient treatment that will have to be reconsidered in the current macroeconomic context.
\end{abstract}

Keywords: Epidemiology; PUVA Therapy; Photobiology; Psoriasis; Time-to-Treatment

\section{INTRODUCTION}

For years, phototherapy has been used in a wide range of skin diseases, which is unsurprising as skin is the anatomical feature most directly exposed to light. Phototherapy became a common practice in dermatology at the beginning of the $20^{\text {th }}$ century, when Goeckerman introduced a combination of coal tar and UVB. This technique would be named after him. Later, Ingram developed a similar treatment protocol, which consisted of a 15-30-minute bath in a tar solution followed by a UVB dose. It started with $30-50 \%$ of the minimum erythema dose - the meaning and use of which will be explained later- and this light dose was increased by $30-50 \%$ at each session. Subsequently, an anthralin paste with low concentrations (0.05$0.1 \%)$ was applied to and maintained on the treated intertegument for 6-24 hours. ${ }^{1}$
As a general rule, phototherapy was only used under special conditions, involving a large progression of psoriasis. Retinoids were then developed, including acitretin, along with immunosupressive drugs like cyclosporine and methotrexate, followed by the modern biologic therapies. Although this has meant that phototherapy is no longer the most widely used treatment for psoriasis, it is still frequently indicated to treat this disease, especially in cases of formal contraindication, pateints' refusal to be treated with any of the previous therapies, or pregnancy.

In recent years, many studies have compared the success of different types of phototherapy in psoriasis treatment. No significant differences were observed in the number of sessions - mainly Broadband UVB, oral PUVA and PUVA bath- nor in the total cumulative exposure. Percentages for whitening effectiveness were similar and the periods of remission did not involve differences in terms of the number of days. ${ }^{2}$ In 2004,

Received on 11.05.2014.

Approved by the Advisory Board and accepted for publication on 25.08.2014.

Work performed at the Hospital Universitario Virgen Macarena - Seville, Spain.

Financial support: None.

Conflicts of Interest: None.

Hospital de Especialidades de Jerez - Jerez de la Frontera, Cádiz. Spain

Universidad de Sevilla - Seville, Spain

Hospital Universitario Virgen Macarena - Seville, Spain.

(C)2015 by Anais Brasileiros de Dermatologia

An Bras D ermatol. 2015;90(4):473-8. 
Tahir et al. compared oral PUVA therapy and Narrow Band UVB in patients with plaque psoriasis. They concluded that PUVA therapy has a higher whitening effectiveness percentage as it requires lower total cumulative exposure and fewer sessions. ${ }^{3}$ However, the British Phototherapy Group suggests that Narrow Band UVB light therapy is slightly less effective than PUVA therapy (strength of recommendation A, evidence level I) for treating psoriasis in patients with skin phototypes I-III, though it requires fewer sessions. Importantly, patients who undergo many phototherapy sessions during childhood need to be periodically examined and must have the minimum number of sessions to control the disease in future treatments. ${ }^{4}$

Nowadays, two different classic phototherapy treatments are recognized in dermatology, excluding new developments such as the $308 \mathrm{~nm}$ excimer laser or other forms of localized phototherapy:

- Phototherapy that uses UVB radiation only. These can be Narrow Band UVB (311-313nm, a modern development) or lamps that cover the whole UVB spectrum (Broadband UVB, developed beforehand)

- Photochemotherapy, which involves a combination of UVA light and a photosensitizer chemical, normally from the family of psoralens. They are administered orally or topically.

Despite the differences between both treatments, many authors agree that there are no significant differences between the oral PUVA treatment and the PUVA bath therapy. However, Narrow Brand UVB is different in that it requires a lower dose for each session and fewer sessions. Thus, it is now the preferred option for treating psoriasis with light therapy. Narrow Band UVB enables lower radiation on the body's surface as the UVB dose is 15-20 times less powerful than that needed for UVA. No systemic adverse effects emerge because it does not require oral or topical administration of psoralens. Furthermore, there is a significant reduction in total cumulative exposure and fewer sessions overall, allowing more time between doses, thus providing more comfort for the patient. The period during which patients do not receive light therapy is known (3-6 months) but no differences regarding the type of light therapy used have been published. 4,5

Frequency of treatment per week is an important and controversial factor in light therapy, often predetermining its compliance. The British Phototherapy Group recommends a frequency of 2 or 4 times a week if the 8-MOP is the chosen psoralen for PUVA, either oral or topical - if there is a control with the oral drug the sessions can be limited to 2 per week - at a frequency of 2 sessions a week with UVB radiation. ${ }^{5}$

\section{METHODS}

We have designed a descriptive study encompassing a univariate analysis covering 443 treatments with light therapy, all administered to the same number of psoriasis patients. The aim was to characterize the patient population that had received the aforementioned treatment in the $V$ irgen $M$ acarena Health Area in Seville (Spain) between June 1985 and October 2011. The following variables were taken into account for each patient that had undergone light therapy: sex, year of birth, diagnosis, phototype, type of radiation received, starting year of treatment, dose number, first dose, last dose, reason why the treatment ended and side effects. A descriptive study of the study variables and a linear regression model were carried out in order to determine the possible mathematical relationship between the total number of sessions and the factors affecting the number of sessions depending on the type of radiation used. Patients who required a second course of treatment were not included in this count. The established total cumulative dose was never exceeded, as this can provoke the development of serious side effects.

The protocol followed to treat patients was the Consensus document on phototherapy: PUVA therapy and narrow-band UVB therapy, published by the Spanish Phototherapy Group in 2005, along with its anterior and further versions. ${ }^{5}$

A descriptive analysis of the study variables was performed for the statistical processing of data. The study variables were divided into quantitative continuous variables, discrete quantitative variables and qualitative variables. For the qualitative variables, a univariate descriptive analysis was performed, calculating the absolute and the cumulative frequency; while for the quantitative variables, both the central tendencies (median and mode) and the dispersion measures (standard deviation) were calculated. Variables were tested for normality through the Saphiro-Wilk test. Statistical analysis was performed with the R program (R Development Core Team, 2011), version 2.14.

\section{RESULTS}

1.General descriptive study

1. 1. Demographic data

A total of 443 patients were studied, of which $52.9 \%$ were men, while $47.1 \%$ were women, as shown in table 1 . The average age was 43 , with a standard deviation of 16.53, whereas the median was lower (42). Table 2 displays data according to the different age ranges.

\section{Reasons why the treatment ended:}

Out of a total number of patients evaluated, 
TABLE 1: Gender distribution

\begin{tabular}{llll}
\hline & Frequency & Percentage & $\begin{array}{l}\text { Cumulative } \\
\text { percentage }\end{array}$ \\
\hline Women (W) & 209 & 47.1 & 47.1 \\
Men (M) & 234 & 52.9 & 100.0 \\
\hline Total & $\mathbf{4 4 3}$ & $\mathbf{1 0 0 . 0}$ & $\mathbf{1 0 0 . 0}$
\end{tabular}

TABLE 2: Age range distribution

\begin{tabular}{llll}
\hline Ranges & Frequency & Percentage & $\begin{array}{l}\text { Cumulative } \\
\text { percentage }\end{array}$ \\
\hline$[6,13.8)$ & 5 & 1.2 & 1.2 \\
{$[13.8,21.6)$} & 41 & 9.3 & 10.5 \\
{$[21.6,29.4)$} & 60 & 13.6 & 24.1 \\
{$[29.4,37.2)$} & 73 & 16.5 & 40.6 \\
{$[37.2,45)$} & 64 & 14.4 & 55.0 \\
{$[45,52.8)$} & 66 & 14.9 & 69.9 \\
{$[52.8,60.6)$} & 54 & 12.1 & 82.0 \\
{$[60.6,68.4)$} & 47 & 10.6 & 92.6 \\
{$[68.4,76.2)$} & 23 & 5.2 & 97.8 \\
{$[76.2,84]$} & 10 & 2.2 & 100.0 \\
\hline Total & $\mathbf{4 4 3}$ & $\mathbf{1 0 0 . 0}$ & $\mathbf{1 0 0 . 0}$ \\
\hline
\end{tabular}

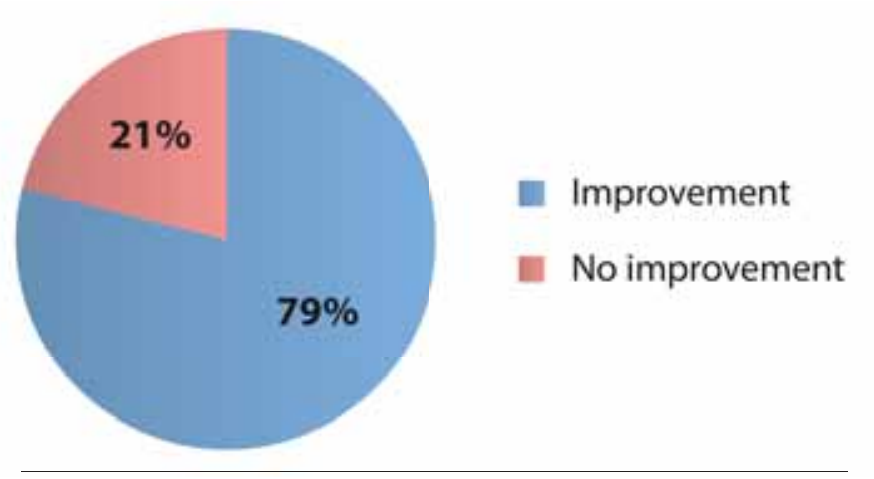

Graph 1: Percentages of improvement (or not) in treated patients.

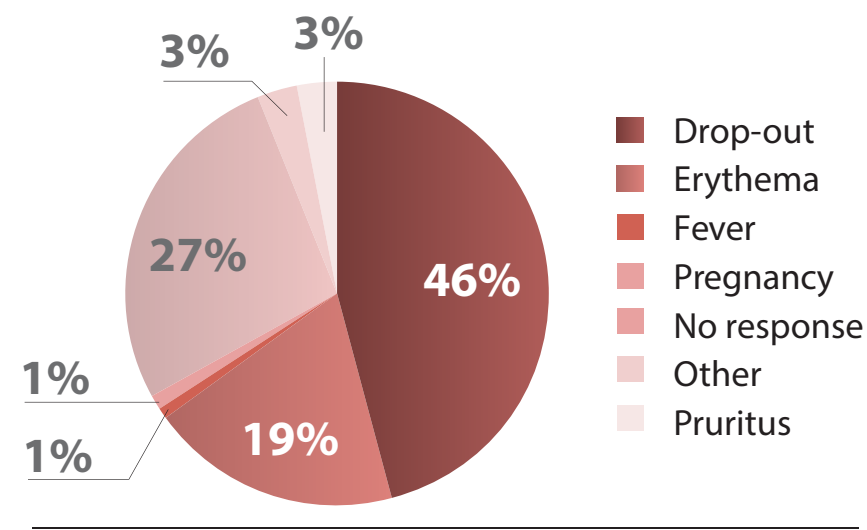

GRAPH 2: Distribution of causes of treatment discontinuation.

TABLE 3: Distribution of patients according to type of radiation received

\begin{tabular}{llll}
\hline Typeofradiation & Frequency & Percentage & Cumulativepercentage \\
\hline PUVA & 77 & 17.3 & 17.3 \\
PUVA+UVB & 46 & 10.4 & 27.7 \\
UVB & 84 & 19.1 & 46.7 \\
Narrow Band UVB & 236 & 53.3 & 100.0 \\
\hline Total & 443 & 100.0 & 100.0 \\
\hline
\end{tabular}

351 patients $(79.15 \%)$ were discharged due to improvement or healing, while 92 patients $(20.85 \%)$ were discharged for other reasons, as demonstrated in graph 1 . Furthermore, graph 2 shows the reasons why this $20.85 \%$ interrupted the treatment.

\section{Type of radiation used}

Table 3 displays patient data according to the different radiations used in their treatment. Most patients were treated with Narrow Band UVB (236 patients, 53.3\%), followed by Broadband UVB treatment (84 patients, 19.1\%), PUVA (77 patients, $17.3 \%$ ), and finally PUVA and Broadband
UVB treatment (46 patients, $10.4 \%$ ). The latter was administered in the early 90s, until the department purchased a light therapy cabin with nbUVB lamps.

\section{Phototype distribution}

Since the distribution corresponds to the geographical area where the study was performed, most patients had phototype III (362 patients, 81, 71\%) followed by phototype II (64 patients, 14, 44\%) and IV (17 patients, 3, 83\%). There were no patients with phototypes V or VI.

We addressed separately the question of whether the average number of sessions needed for 
TABLE 4: Phototype distribution and average dosis

\begin{tabular}{lllll} 
Phototype & N. & Average & Median & $\begin{array}{l}\text { Standard } \\
\text { deviation }\end{array}$ \\
\hline II & 64 & 32.52 & 29.00 & 16.49 \\
III & 362 & 29.97 & 26.00 & 15.55 \\
IV & 17 & 35.12 & 34.00 & 16.96 \\
\hline
\end{tabular}

improvement changed depending on each patient's phototype. Rergarding the total number of sessions for phototype II variable, 64 valid cases were found, with an average of 32.52 units and a standard deviation of 16.49 , whereas the median was lower (29 units). In addition, 362 valid cases were found in patients with phototype III. The average was 29.97 units, with a standard deviation of 15.55 and a median of 26 sessions. Further, 17 valid cases were found for phototype IV, with an average of 35.12 sessions and a standard deviation of 16.96 as well as a median of 34 units. No atypical factors were observed in the sessions (Table 4)

Moreover, we distributed the patients into two groups to estimate the average total cumulative exposure that reached patients who experienced improvement in their psoriasis, with or without treatment. The average for such patients was 33.38 units, with a standard deviation of 14,88 , while the median was lower (30 units). Furthermore, $50 \%$ of the central observations ranged from 21.75 units to 42 units. Patients who showed no improvement (75) received an average total cumulative exposure of 42.46 $\mathrm{J} / \mathrm{cm}^{2}$ (Table 5).

\section{DISASSION}

The mid-1990s witnessed the advent of nb UVB, a revolution in this field enabling treatment of the same diseases and with similar results, but involving a lower dose per session and fewer total sessions. This was possible because the biological effectiveness of the $\mathrm{nb}$ UVB radiation was over 1,000 times higher than that of UVA radiation. ${ }^{6}$ Until then, oral PUVA and PUVA-bath had been equal in terms of therapeutic

TABLE 5: Total cumulative exposure in patients with or without improvement.

\begin{tabular}{lllll}
\hline & N. & Average & Median & $\begin{array}{l}\text { Standard } \\
\text { deviation }\end{array}$ \\
\hline Improvement & 368 & 131.53 & 61.30 & 144.58 \\
No improvement 75 & 42.46 & 12.62 & 84.80 \\
\hline
\end{tabular}

effectiveness. ${ }^{7}$ In addition, the harmlessness of these therapies, at least in the short- and medium-term, has fostered their use in a significant number of cases. Their effectiveness can be proven in many diseases, which has encouraged quasi-experimental indications for diseases in which classical therapies were unsatisfactory.

When determining whether the total number of sessions was affected by the different skin phototypes in psoriasis, it was clear that although most patients had phototype III, the average number of sessions was similar to that for patients with common phototypes (29.97 sessions for phototype III patients versus 32.52 in those with phototype II and 35.12 for phototype IV patients). Therefore, a different phototype not imply an earlier improvement in phototherapy. This had already been proven in the literature, which described guaranteed optimal results in the first 30 sessions of treatment, meaning 6-8 weeks with cumulative doses of $60-150 \mathrm{~J} / \mathrm{cm}^{2} 8,9$

Total number of patients to be discharged as a result of improvement

Out of a total of 443 studied patients, 368 were discharged as a result of improvement. Irrespective of the clinical feature that had prompted the treatment, the average number of sessions needed was $32.34 ; 50 \%$ of these observations were between 21 and 42 sessions The presence of atypical values did not interfere significantly in these results, which can be compared with those obtained from other published series. ${ }^{10,11}$

Total cumulative exposure to be discharged as a result of improvement

When interpreting this variable, the corrected mean value was $131.53 \mathrm{~J} / \mathrm{cm}^{2}$, irrespective of the number of sessions needed to achieve improvement in the disease. Compared with previous studies, this total cumulative exposure is similar or slightly lower. A possible explanation for this is that more than half the patients did not receive PUVA treatment, but rather Narrow Band UVE treatment, which uses lower energies than other cabins. ${ }^{12,13}$ However, other studies show similar data to ours, though just for PUVA. There were no previous studies for Narrow Band UVB on this matter in our area of expertise.

Nevertheless, the value of this variable could be altered by the fact that the more sessions undergone, the higher the total cumulative exposure. With the exception of this particular instance, we have not found any studies revealing a higher response to lower total cumulative exposure. However, some studies demonstrate the same results as those in most publications, with lower average total doses than what is generally accepted. ${ }^{14}$ 


\section{effects}

\section{Therapy drop-out and conditioning side}

As previously mentioned, in order to guarantee a more truthful analysis of the data and in view of the difficulty of gathering all the information about medical histories (some of which werealready closed), the decision was taken to classify the treatment's success according to "medical discharge as a result of improvement" or "dropout". In the latter case, the circumstance that caused the end of treatment was specified. Indeed, $45.75 \%$ of the 92 cases of drop-out were the result of the patient's decision. In some cases, patients did not notice satisfactory results after treatment, while in others, patients were unable to balance treatment with their daily lifestyle. The second cause of drop-out was the ineffectiveness of treatment for the disease treated (27\%). Lastly, the third cause was the appearance of erythema (19\%), although it generally subsided following temporal interruption of UV therapy and was later treated with lower initial radiation doses.

Many publications describe different skin diseases and the success of treatments regarding the psychological well-being of patients, as well as the speed of improvement between the beginning of treatment and successful outcomes. ${ }^{15}$ This is particularly important in light therapy as, although there are pathologies in which response to treatment is excellent (for instance, in certain types of psoriasis like guttate psoriasis, or atopic dermatitis), other diseases are more difficult to treat, which can be attributed to poor response to UV radiation or the chronic nature of the disease.

\section{Oncogenesis}

NMSC: Although no publications substantiate the theory that greater exposure to Narrow Band UVB radiation entails an increase in NMSC among treated populations, the risk of squamous-cell carcinoma in patients treated with PUVA is proportional to total cumulative exposure. This exposure was 11 times higher in patients exposed to over 200 PUVA sessions than in those who received under 100 sessions. ${ }^{16-19}$ Intially, these data can seem cut-rated but it is possible that the patients evaluated in different studies have a different profile concerning light exposure and phototype from that of the patients in our study. Several European studies noted that the behavior of tumors did not differ from their behavior among the general population.

No increase in proportion has been observed in patients who develop basal-cell carcinoma after light therapy treatment, but they undergo the same safety measures as patients with other NMSCs (150-200 sessions).

Melanoma: According to some authors, unlike for NMSC, the melanoma incidence may behigher in patients who received over 250 sessions and were monitored for more than 15 years. ${ }^{20}$ For this reason, we took a random sample of 50 patients from the 254 who underwent light therapy treatment between 1985 and 1995. They enabled us to conduct a monitoring period and we did not find any cases of melanoma in this sample.

\section{Photoaging}

Although few studies have evaluated photoaging in patients treated with light therapy, an increase in light damage can be observed in patients who have received long treatments, as demonstrated both in experimental studies and clinical practice. ${ }^{21-23}$ Hence, it is important to minimize this risk as it occurs with carcinogenesis. We should limit the number of treatments and recommend light protectors to block UV radiation from the environment.

\section{Side effects linked to psoralen intake:}

About a quarter of patients treated with 8-MOP experience gastrointestinal discomfort or nausea within hours of taking the drug. These side effects appear in the literature as major causes of treatment drop-out but no patients in our study who had received treatment with PUVA interrupted treatment for this reason. ${ }^{24}$ In order to reduce these effects, practioners recommend 8-MOP, together with fatty food, or in separate doses.

Many studies mention the hepatotoxiciy that can affect patients treated with 8-MOP, which accounts for a regular, analytical control of transaminases in patients receiving PUVA treatment. This hepatotoxicity can be considered idiosyncratic because 8-MOP has not been shown to entail hepatotoxicity in large studies conducted in the USA and Europe. In these studies, monitoring liver biopsies were even performed and it was boosted for patients already presenting liver disease. Although an analysis of liver function is recommended at the beginning of treatment, the need to monitor is debatable. Some patients also complain of neurological effects such as insomnia, headaches and asthenia after taking this substance but these effects usually subside after a reduction in the dose.

\section{CONCUSIONS}

Although the role of light therapy has been replaced by different therapeutic modalities in recent years -especially in psoriasis-, this treatment has become established as an option for many skin diseases. From the outset, it has been regarded as a particularly effective treatment for psoriasis, as previously mentioned, leading to similar results irrespective of the type of radiation used. Furthermore, it is an effective and efficient treatment that will have to be reconsidered in the current macroeconomic context. $\square$ 


\section{REFERENCES}

1. Lee E, Koo J. Modern modified "ultra” Goeckerman therapy: a PASI assessment of a very effective therapy for psoriasis resistant to both prebiologic and biologic therapies. J Dermatolog Treat. 2005;16:102-7.

2. Duarte I, Cunha JA, Bedrikow RB, Lazzarini R. What is the most common phototherapy prescription for psoriasis: NB-UVB or PUVA? Prescription behavior. An Bras Dermatol. 2009;84:244-8.

3. Tahir R, Mujtaba G. Comparative efficacy of psoralen - UVA photochemotherapy versus narrow band UVB phototherapy in the treatment of psoriasis. J Coll Physicians Surg Pak. 2004;14:593-5.

4. Tan E, Lim D, Rademaker M. Narrowband UVB phototherapy in children: A New Zealand experience. Australas. Australas J Dermatol. 2010;51:268-73.

5. Carrascosa JM1, Gardeazábal J, Pérez-Ferriols A, Alomar A, Manrique P, JonesCaballero $\mathrm{M}$, et al. Consensus document on phototherapy: PUVA therapy and narrow-band UVB therapy. Actas Dermosifiliogr. 2005;96:635-58.

6. Walters IB1, Burack LH, Coven TR, Gilleaudeau P, Krueger JG. Suberythematogenic narrow-band UVB is markedly more effective than conventional UVB in treatment of psoriasis vulgaris. J Am Acad Dermatol. 1999;40:893-900.

7. Rodríguez-Granados MT, Carrascosa JM, Gárate T, Gómez-Díez S, GuimaraensJuantorena $D$. Documento de consenso sobre la modalidad terapéutica del baño PUVA. Actas Dermosifiliogr. 2007;98:164-70.

8. Gonzalez E. PUVA for psoriasis. Dermatol Clin. 1995;13:851-66.

9. Cooper EJ, Herd RM, Priestley GC, Hunter JA. A comparison of bathwater and oral delivery of 8-methoxypsoralen in PUVA therapy for plaque psoriasis. Clin Exp Dermatol. 2000;25:111-4.

10. Lowe N, Weingarten D, Bourget T, Moy L. PUVA therapy for psoriasis: Comparison of oral and bath-water delivery of 8- methoxypsoralen. J Am Acad Dermatol. 1986;14:754-60.

11. Collins P, Rogers S. Bath-water compared with oral delivery of 8-methoxypsoralen PUVA therapy for chronic plaque psoriasis. Br J Dermatol. 1992;127:392-5.

12. Karrer S, Eholzer C, Ackermann G, Landthaler M, Szeimies RM. Phototherapy of psoriasis: comparative experience of different phototherapeutic approaches. Dermatology. 2001;202:108-15.

13. Augustin M, Holland B, Dartsch D, Langenbruch A, Radtke MA. Adherence in the treatment of psoriasis: a systematic review. Dermatology. 2011;222:363-74.

14. Martin SL, McGoey ST, Bebo BF Jr, Feldman SR. Patients' educational needs about topical treatments for psoriasis. J Am Acad Dermatol. 2013;68:e163-8.

15. Thorneloe RJ, Bundy C, Griffiths CE, Ashcroft DM, Cordingley L. Adherence to medication in patients with psoriasis: a systematic literature review. Br J Dermatol. 2013;168:20-31.

16. Stern RS, Laird N, Melski J, Parrish JA, Fitzpatrick TB, Bleich HL.Cutaneous squamous-cell carcinoma in patients treated with PUVA. N Engl $\mathrm{J}$ Med. 1984;310:1156-61.

17. Weischer M, Blum A, Eberhard F, Röcken M, Berneburg M. No evidence for increased skin cancer risk in psoriasis patients treated with broadband or narrowband UVB phototherapy: A first retrospective study. Acta Derm Venereol. 2004;84:370-4.

18. Sano T, Kume T, Fujimura T, Kawada H, Higuchi K, Iwamura M, et al. Long-term alteration in the expression of keratins 6 and 16 in the epidermis of mice after chronic UVB exposure. Arch Dermatol Res. 2009;301:227-37.
19. Luo D, Min W, Lin XF, Wu D, Xu Y, Miao X. Effect of epigallocatechingallate on ultraviolet B-induced photo-damage in keratinocyte cell line. Am J Chin Med. 2006;34:911-22.

20. Stern RS, Nichols KT, Väkevä LH. Malignant melanoma in patients treated for psoriasis with methoxalen and ultraviolet A radiation (PUVA). N Engl $\mathrm{J}$ Med. 1997;336:1041-5.

21. Tilkorn DJ, Schaffran A, Al-Benna S, Hauser J, Steinau HU, Ring A. Severe Burn Injuries Induced by PUVA Chemotherapy. J Burn Care Res. 2013;34:e195-200.

22. Bretterklieber A, Legat FJ, Wolf P, Hofer A. Retrospective long-term follow-up in patients with chronic palmoplantar dermatoses after good response to bath PUVAtherapy. J Dtsch Dermatol Ges. 2012;10:814-8.

23. Carrascosa JM, Rodríguez Caruncho C. Efectos adversos de la fototerapia: clínica y manejo. Piel. 2008;23:447-52.

24. Garcia-Doval I, Carretero G, Vanaclocha F, Ferrandiz C, Daudén E, Sánchez-Carazo $\mathrm{JL}$, et al. Risk of serious adverse events associated with biologic and nonbiologic psoriasis systemic therapy: patients ineligible vs eligible for randomized controlled trials. Arch Dermatol. 2012;148:463-70.

How to cite this article: Toledo-Pastrana T, García-Hernández MJ, Carrizosa-Esquivel AM, Camacho-Martínez FM. Evaluation of 25 years of phototherapy for treating psoriasis at a teaching hospital in southern Spain. An Bras Dermatol. 2015;90(4):473-8. 